الترقيم الدولي ـ9 Email: utjsci@utq.edu.iq

website: http://jsci.utq.edu.iq

\title{
The Finite Element Method for Nonlinear Huxely Equation
}

\author{
Ahmed Jabbar Hussein \\ Department of Mathematics - College of Education - University of Thi-Qar
}

\begin{abstract}
In this paper, we present a finite element method (F.E.M.) for solving the non-linear huxely equation by using Crank-Nicolson scheme with the predictor - corrector method. The numerical results and absolute errors showed that the Crank - Nicolson finite element method is more accurate and better than respectively from the numerical results and the absolute errors which that presented by Explicit and Crank-Nicolson finite difference method (F.D.M.) [7] .
\end{abstract}

Keyword: Finite element, Huxely equation, Crank-Nicolson

\section{Introduction}

From nonlinear diffusion equations the Generalized burgers huxely equation

$$
\begin{aligned}
& \frac{\partial u}{\partial t}+\alpha u^{\delta} \frac{\partial u}{\partial x}-\frac{\partial^{2} u}{\partial x^{2}}=\beta u\left(1-u^{\delta}\right)\left(u^{\delta}-a\right) \\
& \alpha \geq 0, \beta \geq 0, \delta>0, \text { and } a \in(0,1)
\end{aligned}
$$

Equation (1) is extension formula for burgers huxely - equation. When $\alpha \neq 0, \beta \neq 0$ and $\delta=1$, The above equation transform to burgers huxely equation:

$$
\frac{\partial u}{\partial t}+\alpha u \frac{\partial u}{\partial x}-\frac{\partial^{2} u}{\partial x^{2}}=\beta u(1-u)(u-a) .
$$

Equation (2) is prime model to describe the relation between the reaction procedure and effectives the solution and diffusion. Equation (1) has special two cases are burgers and huxely equation, When $\delta=1$ and $\beta=0$ equation (1) transform to the burgers equation

$$
\frac{\partial u}{\partial t}+\alpha u \frac{\partial u}{\partial x}-\frac{\partial^{2} u}{\partial x^{2}}=0
$$

Which describe the far domain to wave diffusion in dissipative dynamical systems, When $\delta=1$ and $\alpha=0$ equation (1) transform to huxely equation 
$\frac{\partial u}{\partial t}-\frac{\partial^{2} u}{\partial x^{2}}=\beta u(1-u)(u-a)$ in $\Omega \times[0, \infty)$

$0<a<1, \beta>0,-1 \leq x \leq 1$, and $t \geq 0$

with inital condition and boundary condition

$$
\begin{aligned}
& u(x, 0)=(b-H) x^{2}+H \\
& 0 \leq b \leq 1, \text { and } 0<H \leq 1, \\
& u(-1, t)=u(1, t)=b
\end{aligned}
$$

Which describe the diffusion the nervous impulses in nervous fibers and wall motion in liquid crystals . The effect of constant $H$ on the numerical solution is made the solution curve either convex (the maximum value to solution at $\mathrm{x}=0$ ) or concave (the minimum value to the solution at $\mathrm{x}=0$ ).

Equations (3) and (4) have important rules in nonlinear physical, because these equations important in the nonlinear phenomena's study .

Frank and Zeldovich [3] (1938) introduced equation (4) as model to study diffusion the nervous impulses, they proved that the transformation wave has the figure and the Velocity as follows :

$$
u(x, t)=\frac{1}{1+\exp [(x-v t) / \sqrt{2}]}, \quad v=\frac{1-2 a}{\sqrt{2}}
$$

Maginu [4] (1978) studied the stability of steady state solutions for this type from the diffusion equations. He using the Liapunov method to find the stability condition and he show that this condition connected with Existence condition for these solutions.

Manorenjan [6] (1984) studied in detail the steady state case $u=a$, he used the pseudospectral method and Fortran IV language for this proposed .

Binczak, Eilbeck and Scort [1] (2001) using this equation as model for operating nervous transducer (conducted), they using the numerical results (by using predictor-corrector method) to expression this operation.

Mohammad abd [7] (2005) studied stationary stability and numerical solution for this equation by using stability analysis type Fourier (von Neumann). Also he solve this equation numerically in two methods from finite difference methods, the first is the Explicit finite difference method, the second is the Crank - Nicolson finite difference method.

In this paper, huxely equation was solved by the Crank - Nicolson finite element method with predictor - corrector method .The numerical results are compared with the numerical results [7]

The stationary solution for Huxely equation (4) is (see [5] ) 


$$
u(x)=\left[\begin{array}{cc}
3 a / \sqrt{(2-a)(1 / 2-a)} \cosh (\sqrt{a} x)+1+a & 0<a<1 / 2, \quad \beta=1 \\
\frac{1}{2}+\alpha \operatorname{sn}\left(\frac{x}{\sqrt{2}}\left(1 / 2-\alpha^{2}\right)^{1 / 2}, \alpha /\left(1 / 2-\alpha^{2}\right)^{1 / 2}\right), & a=1 / 2, \beta=1, \quad 0<\alpha<1
\end{array}\right.
$$

Where $s n(v, d)$ represent the Jacobi Elliptic Function for argument $\mathrm{v}$ and the modulus $\mathrm{d}$, The Taylor series of function $\operatorname{sn}(v, d)$ is (see [2]) :

$$
\operatorname{sn}(v, d)=v-\left(1+d^{2}\right) \frac{v^{3}}{3 !}+\left(1+14 d^{2}+d^{4}\right) \frac{v^{5}}{5 !}-\left(1+135 d^{2}+135 d^{4}+d^{6}\right) \frac{v^{7}}{7 !}+\ldots
$$

\section{The Finite Element Method}

The domain $[-1,1] \times[0, r]$ is divided into $M_{x} \times N_{t}$ mesh with the spatial step size $\Delta x=h=(1-(-1)) / M_{x}=2 / M_{x}$ and the time size $\quad \Delta t=k=T / N_{t} \cdot$, we denote $U(m \Delta x, n \Delta t)$, conveniently, by $U_{m}^{n}$ and the nodal points $\left(x_{m}, t_{n}\right)$ are given by $x_{m}=x_{0}+m h, t_{n}=n k$.

Remark: The following differentiation and integration results hold over the element (e):(see [8])

(i) $\frac{\partial N_{m-1}}{\partial x}=-\frac{1}{x_{m}-x_{m-1}}=-\frac{1}{h}, \frac{\partial N_{m}}{\partial x_{m}}=\frac{1}{x_{m-1}-x_{m-1}}=\frac{1}{h}$

(ii)

$$
\int_{m-1}^{x_{m}} N_{m-1}^{r} N_{m}^{t} d x=\frac{r ! t !\left(x_{m}-x_{m-1}\right)}{(r+t+1) !}
$$

Where $r$ and $t$ are positive integers.

We use the linear piecewise approximation in the space variable and Galerkin method to obtain the semi - discrete approximation to (4)

we have

$$
U^{(e)}=N_{m-1}(x) U_{m-1}(t)+N_{m}(x) U_{m}(t)
$$

where

$$
N_{m-1}=\frac{x_{m}-x}{h^{(e)}}, N_{m}=\frac{x-x_{m-1}}{h^{(e)}}, h^{(e)}=x_{m}-x_{m-1}
$$

we also have

$$
\frac{\partial U}{\partial t}=N_{m-1} \frac{d U_{m-1}}{d t}+N_{m} \frac{d U_{m}}{d t}
$$

Galerkin equations in matrix form may be written as : 


$$
\begin{aligned}
& \int_{m-1}^{x_{m}}\left\{\left[\begin{array}{cc}
N_{m-1} N_{m-1} & N_{m-1} N_{m} \\
N_{m} N_{m-1} & N_{m} N_{m}
\end{array}\right]\left[\begin{array}{c}
U_{m-1}^{\cdot} \\
U_{m}^{\cdot}
\end{array}\right]+\left[\begin{array}{cc}
N_{m-1}^{\prime} N_{m-1}^{\prime} & N_{m-1}^{\prime} N_{m}^{\prime} \\
N_{m}^{\prime} N_{m-1}^{\prime} & N_{m}^{\prime} N_{m}^{\prime}
\end{array}\right]\left[\begin{array}{c}
U_{m-1} \\
U_{m}
\end{array}\right]+\right. \\
& {\left[\begin{array}{l}
N_{m-1}\left(N_{m-1} U_{m-1}+N_{m} U_{m}\right)^{3} \\
N_{m}\left(N_{m-1} U_{m-1}+N_{m} U_{m}\right)^{3}
\end{array}\right]-(1-a)\left[\begin{array}{l}
N_{m-1}\left(N_{m-1} U_{m-1}+N_{m} U_{m}\right)^{2} \\
N_{m}\left(N_{m-1} U_{m-1}+N_{m} U_{m}\right)^{2}
\end{array}\right]+} \\
& \left.\quad\left[\begin{array}{cc}
N_{m-1} N_{m-1} & N_{m-1} N_{m} \\
N_{m-1} N_{m-1} & N_{m} N_{m}
\end{array}\right]\left[\begin{array}{c}
U_{m-1} \\
U_{m-1}
\end{array}\right]\right\} d x=0
\end{aligned}
$$

By using the remark ( $\mathrm{i}$ and ii) we have

$$
\begin{aligned}
& \frac{h}{6}\left[\begin{array}{ll}
2 & 1 \\
1 & 2
\end{array}\right]\left[\begin{array}{c}
U_{m-1}^{\cdot} \\
U_{m}^{\cdot}
\end{array}\right]+\frac{1}{h}\left[\begin{array}{cc}
1 & -1 \\
-1 & 1
\end{array}\right]\left[\begin{array}{c}
U_{m-1} \\
U_{m}
\end{array}\right]+ \\
& {\left[\begin{array}{c}
N_{m-1}^{4} U_{m-1}^{3}+3 N_{m-1}^{3} N_{m} U_{m-1}^{2} U_{m}+3 N_{m-1}^{2} N_{m}^{2} U_{m-1} U_{m}^{2}+N_{m-1} N_{m}^{3} U_{m}^{3} \\
N_{m-1}^{3} N_{m} U_{m-1}^{3}+3 N_{m-1}^{2} N_{m}^{2} U_{m-1}^{2} U_{m-1}+3 N_{m-1} N_{m}^{3} U_{m-1} U_{m}^{2}+N_{m}^{4} U_{m}^{3}
\end{array}\right]-} \\
& (1+a)\left[\begin{array}{l}
N_{m-1}^{3} U_{m-1}^{2}+2 N_{m-1}^{2} N_{m} U_{m-1} U_{m}^{+N_{m-1}} N_{m}^{2} U_{m}^{2} \\
N_{m-1}^{2} N_{m} U_{m-1}^{2}+2 N_{m-1} N_{m}^{2} U_{m-1} U_{m}+N_{m}^{3} U_{m}^{2}
\end{array}\right]+\frac{a h}{6}\left[\begin{array}{ll}
2 & 1 \\
1 & 2
\end{array}\right]\left[\begin{array}{c}
U_{m-1} \\
U_{m}
\end{array}\right]=0
\end{aligned}
$$

by rearranging 


$$
\begin{aligned}
& \frac{h}{6}\left[\begin{array}{ll}
2 & 1 \\
1 & 2
\end{array}\right]\left[\begin{array}{c}
U_{m-1} \\
\dot{U_{m}}
\end{array}\right]+\frac{1}{h}\left[\begin{array}{cc}
1 & -1 \\
-1 & 1
\end{array}\right]\left[\begin{array}{c}
U_{m-1} \\
U_{m}
\end{array}\right]+\left[\begin{array}{l}
\frac{h}{5} U_{m-1}^{3}+\frac{3 h}{20} U_{m-1}^{2} U_{m}+\frac{h}{10} U_{m-1} U_{m}^{2}+\frac{h}{20} U_{m}^{3} \\
\frac{h}{20} U_{m-1}^{3}+\frac{h}{10} U_{m-1}^{2} U_{m}+\frac{3 h}{20} U_{m-1} U_{m}^{2}+\frac{h}{5} U_{m}^{3}
\end{array}\right]- \\
& (1+a)\left[\begin{array}{l}
\frac{h}{4} U_{m-1}^{2}+\frac{h}{6} U_{m-1} U_{m}+\frac{h}{12} U_{m}^{2} \\
\frac{h}{12} U_{m-1}^{2}+\frac{h}{6} U_{m-1} U_{m}+\frac{h}{4} U_{m}^{2}
\end{array}\right]+\frac{a h}{6}\left[\begin{array}{cc}
2 & 1 \\
1 & 2
\end{array}\right]\left[\begin{array}{c}
U_{m-1} \\
U_{m}
\end{array}\right]=0
\end{aligned}
$$

This implies

$$
\begin{aligned}
& \frac{h}{6}\left[\begin{array}{ll}
2 & 1 \\
1 & 2
\end{array}\right]\left[\begin{array}{c}
U_{m-1}^{\cdot} \\
U_{m}^{*}
\end{array}\right]+\frac{1}{h}\left[\begin{array}{cc}
1 & -1 \\
-1 & 1
\end{array}\right]\left[\begin{array}{c}
U_{m-1} \\
U_{m}
\end{array}\right]+\frac{h}{20}\left[\begin{array}{c}
4 U_{m-1}^{3}+3 U_{m-1}^{2} U_{m}+2 U_{m-1} U_{m}^{2}+U_{m}^{3} \\
U_{m-1}^{3}+2 U_{m-1}^{2} U_{m}+3 U_{m-1} U_{m}^{2}+4 U_{m}^{3}
\end{array}\right]- \\
& \frac{(1+a) h}{12}\left[\begin{array}{c}
3 U_{m-1}^{2}+2 U_{m-1} U_{m}+U_{m}^{2} \\
U_{m-1}^{2}+2 U_{m-1} U_{m}+3 U_{m}^{2}
\end{array}\right]+\frac{a h}{6}\left[\begin{array}{cc}
2 & 1 \\
1 & 2
\end{array}\right]\left[\begin{array}{c}
U_{m-1} \\
U_{m}
\end{array}\right]=0
\end{aligned}
$$

We write the element equations for the elements $x_{m-1} \leq x \leq x_{m}$ and $x_{m} \leq x \leq x_{m+1}$ and assemble these element equations, we obtain 


$$
\begin{aligned}
& \frac{h}{6}\left[\begin{array}{ccc}
2 & 1 & 0 \\
1 & 4 & 1 \\
0 & 1 & 2
\end{array}\right]\left[\begin{array}{c}
\cdot \\
U_{m-1}^{\cdot} \\
U_{m} \\
\cdot \\
U_{m+1}
\end{array}\right]+\frac{1}{h}\left[\begin{array}{ccc}
1 & -1 & 0 \\
-1 & 2 & -1 \\
0 & -1 & 1
\end{array}\right]\left[\begin{array}{c}
U_{m-1} \\
U_{m} \\
U_{m+1}
\end{array}\right]+
\end{aligned}
$$

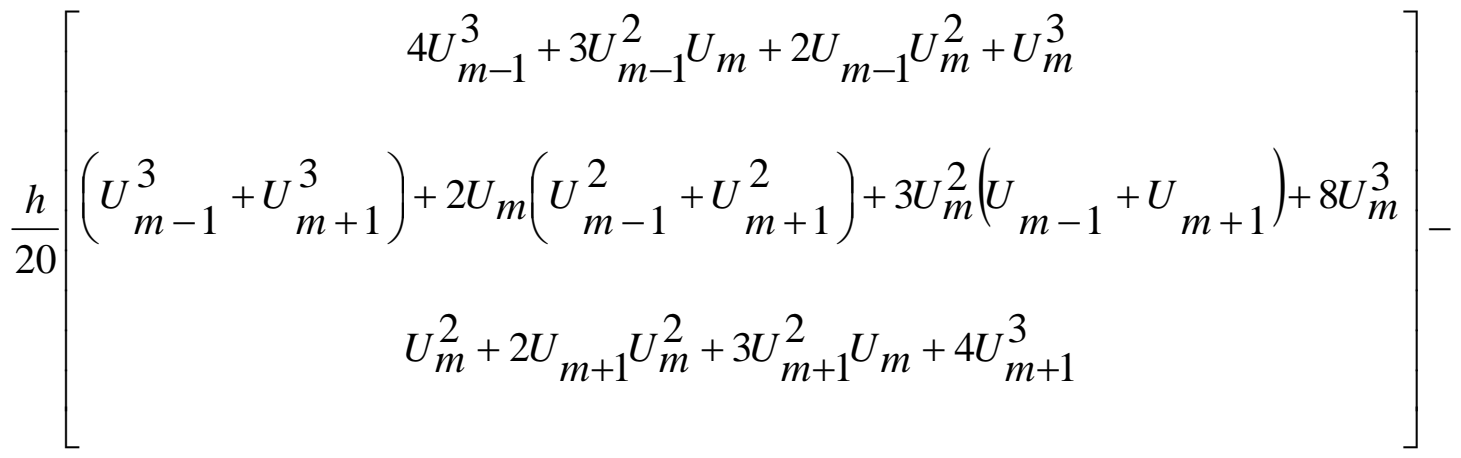

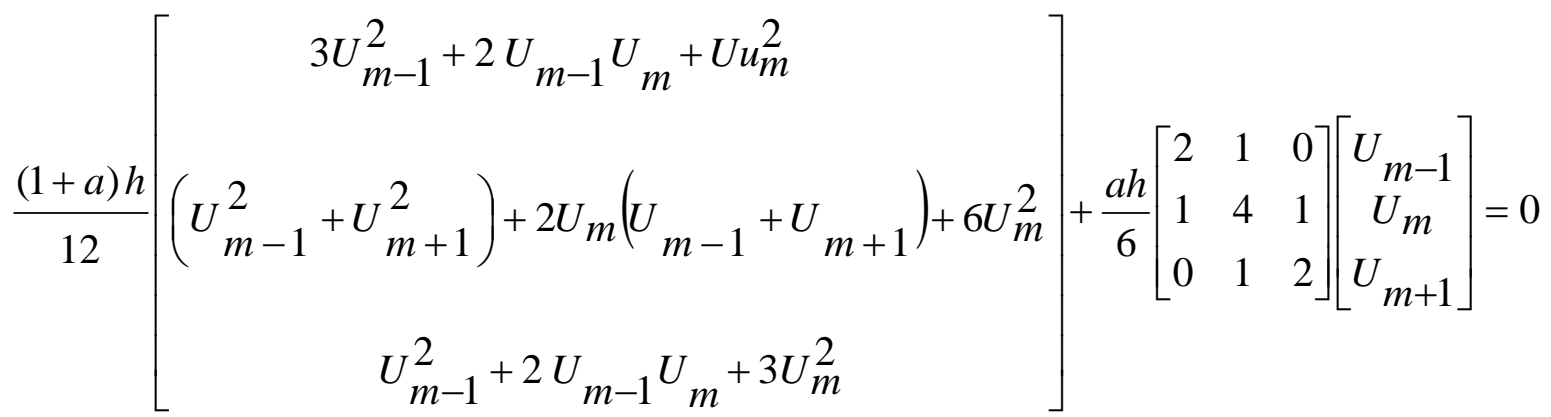

Assembling the element equations and setting the row corresponding to $U_{m}$ to zero. We write the difference - differential equation at the node $x_{m}$ as :

$$
\begin{aligned}
& \frac{h}{6}\left(U_{m-1}^{\cdot}+4 U_{m}+U_{m+1}^{\cdot}\right)+\frac{1}{h}\left(-U_{m-1}+2 U_{m}-U_{m+1}\right)+ \\
& \frac{h}{20}\left(\left(U_{m-1}^{3}+U_{m+1}^{3}\right)+2 U_{m}\left(U_{m-1}^{2}+U_{m+1}^{2}\right)+3 U_{m}^{2}\left(U_{m-1}+U_{m+1}\right)+8 U_{m}^{3}\right)- \\
& \frac{(1+a) h}{12}\left(\left(U_{m-1}^{2}+U_{m+1}^{2}\right)+2 U_{m}\left(U_{m-1}+U_{m+1}\right)+6 U_{m}^{2}\right)+\frac{a h}{6}\left(U_{m-1}+4 U_{m}+U_{m+1}\right)=0
\end{aligned}
$$

Eq.(6) can be written as

$\frac{h}{6}\left(U_{m-1}^{\cdot}+4 \dot{U}_{m}+U_{m+1}^{\cdot}\right)-\frac{1}{h}\left(U_{m-1}-2 U_{m}+U_{m+1}\right)=f_{m}$,

where

$f_{m}=r_{m}+w_{m}+s_{m}$

and 


$$
\begin{aligned}
& w_{m}=-\frac{h}{20}\left(\left(U_{m-1}^{3}+U_{m+1}^{3}\right)+2 U_{m}\left(U_{m-1}^{2}+U_{m+1}^{2}\right)+3 U_{m}^{2}\left(U_{m-1}+U_{m+1}\right)+8 U_{m}^{3}\right), \\
& s_{m}=\frac{(1+a) h}{12}\left(\left(U_{m-1}^{2}+U_{m+1}^{2}\right)+2 U_{m}\left(U_{m-1}+U_{m+1}\right)+6 U_{m}^{2}\right) \\
& r_{m}=-\frac{a h}{6}\left(U_{m-1}+4 U_{m}+U_{m+1}\right),
\end{aligned}
$$

for a positive integer $N_{t}$, let $\Pi_{t}=\left\{t_{n}\right\}_{n=0}^{N_{t}}$ be a partition of $[0, \mathrm{~T}]$ and let $t_{n+1 / 2}=t_{n}+k / 2$, for a function $U$ defined on $\Pi_{t}$,

Let

$$
\dot{U}_{m}=\frac{U_{m}^{n}-U_{m}^{n-1}}{k}, \quad U_{m}^{n+1 / 2}=\frac{U_{m}^{n}+U_{m}^{n-1}}{2}, \quad \delta_{x}^{2}=U_{m-1}-2 U_{m}+U_{m+1}
$$

The Crank - Nicolson Galerkin scheme for the Elliptic problem (4)

$$
h\left(1+\frac{1}{6} \delta_{x}^{2}\right)\left(U_{m}^{n}-U_{m}^{n-1}\right)-\frac{k}{h} \delta_{m}^{2} U_{m}^{n+1 / 2}=k f\left(U_{m}^{n+1 / 2}\right), n=1,2, \ldots . ., N_{t}-1
$$

In matrix form

$$
\left(A+\frac{1}{2} k B\right) U_{m}^{n}=\left(A-\frac{1}{2} k B\right) U_{m}^{n-1}+k f\left(U_{m}^{n+1 / 2}\right)
$$

Where the matrices $\mathrm{A}$ and $\mathrm{B}$ are

$$
A=\frac{h}{6}\left[\begin{array}{ccccccc}
2 & 1 & . & . & . & . & . \\
1 & 4 & 1 & . & . & . & . \\
\cdot & \times & \times & \times & . & . & . \\
\cdot & . & \times & \times & \times & . & . \\
\cdot & . & . & \times & \times & \times & . \\
\cdot & . & . & . & 1 & 4 & 1 \\
\cdot & . & . & . & . & 1 & 2
\end{array}\right], B=\frac{1}{h}\left[\begin{array}{ccccccc}
1 & -1 & . & . & . & . & . \\
-1 & 2 & -1 & . & . & . & . \\
\cdot & \times & \times & \times & . & . & . \\
\cdot & . & \times & \times & \times & . & . \\
. & . & . & \times & \times & \times & . \\
. & . & . & . & -1 & 2 & -1 \\
. & . & . & . & . & -1 & 1
\end{array}\right]
$$

where $(\times)$ and (.) denote a number and an empty location respectively. The matrices A and B are a tridiagonal symmetric matrices.

We observe that as $n=1$ since the equation (7) contain $U^{1}$ in the right side and we have to supplement it with another method for determine $U^{1}$ as initial value. This method will require a separate prescription for calculating $U^{1}$, we shall analyze here a predictor - corrector method for this purpose, using as a first approximation, the value $U^{1}$ determined by the case $\mathrm{n}=1$ of 
equation (7) with $f\left(\frac{U_{m}^{1}+U_{m}^{0}}{2}\right)$ replaced by $f\left(U_{m}^{0}\right)$ and then as the final approximation the result of the same equation with $f\left(\frac{U_{m}^{1}+U_{m}^{0}}{2}\right)$.

i.e.

$$
\begin{aligned}
& \left(A+\frac{1}{2} k B\right) U_{m}^{n}=\left(A-\frac{1}{2} k B\right) U_{m}^{n-1}+k f\left(U_{m}^{n-1}\right) \ldots \ldots \ldots . .(\text { predictor method }) \\
& \left(A+\frac{1}{2} k B\right) U_{m}^{n}=\left(A-\frac{1}{2} k B\right) U_{m}^{n-1}+k f\left(U_{m}^{n+1 / 2}\right) \ldots \ldots \ldots .(\text { correcto method })
\end{aligned}
$$

\section{$\underline{\text { Numerical results }}$}

In this section we obtain a numerical solution of Burgers equation in the form (4), To show the efficiency of the present method for our problem in comparison with the finite difference method [7] we report absolute error which is defined by:

$$
|E|=\left|u\left(x_{i}\right)-U_{m}^{n}\left(x_{i}, t\right)\right|, i, j=1,2,3, \ldots \ldots . . N_{x}-1
$$

In the point $\left(x_{i}, t_{j}\right)$ where $U_{m}^{n}\left(x_{i}, t_{j}\right)$ is the solution obtained by equation (9) solved by finite element method and $u\left(x_{i}\right)$ is stationary solution obtained by equation (5). The code was written in matlabe power station 7 programming language.

We see that when the boundry condition $\mathrm{b}$ take the values $1, \mathrm{a}, 0$ the numerical solution by Crank-Nicolson finite element method and Explicit, Crank-Nicolson finite difference method are converge to the stationary solution $\mathrm{u}=1, \mathrm{u}=\mathrm{a}$ and $\mathrm{u}=0$, respectively with increasing the time, and the our numerical solutions and the absolute errors are more accurate and smaller than, respectively, those of the numerical results by [7] .

The results of Numerical solution and absolute errors by (F.D.M.) and (F.E.M.) are listed in tables 1,2 and 3 with the figures(1-6) which express this results. 
Table 1 : we chose $a=0.3, b=1, H=0.2, \Delta t=0.1$

\begin{tabular}{|c|c|c|c|c|c|c|}
\hline & \multicolumn{4}{|c|}{$\begin{array}{l}\text { The finite difference metho d } \\
\text { [7] }\end{array}$} & \multicolumn{2}{|c|}{$\begin{array}{l}\text { The finite element } \\
\text { method }\end{array}$} \\
\hline $\bar{t}$ & Explicit & $\begin{array}{l}\text { Absolute } \\
\text { error }\end{array}$ & $\begin{array}{l}\text { Crank- } \\
\text { Nicolson }\end{array}$ & $\begin{array}{c}\text { Absolute } \\
\text { enror }\end{array}$ & $\begin{array}{l}\text { Crank- } \\
\text { Nicolson }\end{array}$ & $\begin{array}{c}\text { Absolute } \\
\text { enror }\end{array}$ \\
\hline 0 & 0.4000 & 0.6000 & 0.4000 & 0.6000 & 0.4000 & 0.6000 \\
\hline 0.1 & 0.5624 & 0.4376 & 0.5378 & 0.4622 & 0.6048 & 0.3952 \\
\hline 0.2 & 0.6623 & 0.3377 & 0.6406 & 0.3594 & 0.7302 & 0.2698 \\
\hline 0.3 & 0.7497 & 0.2503 & 0.7216 & 0.2784 & 0.8171 & 0.1829 \\
\hline 0.4 & 0.8144 & 0.1856 & 0.7862 & 0.2138 & 0.8761 & 0.1239 \\
\hline 0.5 & 0.8649 & 0.1351 & 0.8372 & 0.1628 & 0.9162 & 0.0838 \\
\hline 0.6 & 0.9024 & 0.0976 & 0.8772 & 0.1228 & 0.9434 & 0.0566 \\
\hline 0.7 & 0.9303 & 0.0697 & 0.9080 & 0.0920 & 0.9618 & 0.0382 \\
\hline 0.8 & 0.9505 & 0.0495 & 0.9315 & 0.0685 & 0.9742 & 0.0258 \\
\hline 0.9 & 0.9651 & 0.0349 & 0.9493 & 0.0507 & 0.9826 & 0.0174 \\
\hline 1 & 0.9754 & 0.0246 & 0.9627 & 0.0373 & 0.9883 & 0.0117 \\
\hline 1.1 & 0.9828 & 0.0172 & 0.9726 & 0.0274 & 0.9921 & 0.0079 \\
\hline 1.2 & 0.9880 & 0.0120 & 0.9799 & 0.0201 & 0.9947 & 0.0053 \\
\hline 1.3 & 0.9916 & 0.0084 & 0.9853 & 0.0147 & 0.9964 & 0.0036 \\
\hline 1.4 & 0.9941 & 0.0059 & 0.9893 & 0.0107 & 0.9976 & 0.0024 \\
\hline 1.5 & 0.9959 & 0.0041 & 0.9922 & 0.0078 & 0.9984 & 0.0016 \\
\hline 1.6 & 0.9972 & 0.0028 & 0.9943 & 0.0057 & 0.9989 & 0.0011 \\
\hline 1.7 & 0.9980 & 0.0020 & 0.9958 & 0.0042 & 0.9993 & 0.0007 \\
\hline 1.8 & 0.9986 & 0.0014 & 0.9970 & 0.0030 & 0.9995 & 0.0005 \\
\hline 1.9 & 0.9990 & 0.0010 & 0.9978 & 0.0022 & 0.9997 & 0.0003 \\
\hline 2 & 0.9993 & 0.0007 & 0.9985 & 0.0015 & 0.9998 & 0.0002 \\
\hline
\end{tabular}


Table 2 : we chose $a=0.25, b=0.25, H=0.1, \Delta t=0.1$

\begin{tabular}{|c|c|c|c|c|c|c|}
\hline & \multicolumn{4}{|c|}{ The finite difference method [7] } & \multicolumn{2}{|c|}{ The finite element method } \\
\hline $\mathbf{t}$ & Explicit & $\begin{array}{c}\text { Absolute } \\
\text { error }\end{array}$ & $\begin{array}{c}\text { Crank- } \\
\text { Nicolson }\end{array}$ & $\begin{array}{c}\text { Absolute } \\
\text { error }\end{array}$ & $\begin{array}{c}\text { Crank- } \\
\text { Nicolson } \\
\end{array}$ & $\begin{array}{c}\text { Absolute } \\
\text { enror }\end{array}$ \\
\hline 0 & 0.1000 & 0.1500 & 0.1000 & 0.1500 & 0.1000 & 0.1500 \\
\hline 0.1 & 0.1287 & 0.1213 & 0.1274 & 0.1226 & 0.1441 & 0.1059 \\
\hline 0.2 & 0.1573 & 0.0927 & 0.1513 & 0.0987 & 0.1779 & 0.0721 \\
\hline 0.3 & 0.1771 & 0.0729 & 0.1706 & 0.0794 & 0.2007 & 0.0493 \\
\hline 0.4 & 0.1933 & 0.0567 & 0.1862 & 0.0638 & 0.2163 & 0.0337 \\
\hline 0.5 & 0.2056 & 0.0444 & 0.1987 & 0.0513 & 0.2270 & 0.0230 \\
\hline 0.6 & 0.2152 & 0.0348 & 0.2087 & 0.0413 & 0.2343 & 0.0157 \\
\hline 0.7 & 0.2228 & 0.0272 & 0.2167 & 0.0333 & 0.2392 & 0.0108 \\
\hline 0.8 & 0.2287 & 0.0213 & 0.2232 & 0.0268 & 0.2426 & 0.0074 \\
\hline 0.9 & 0.2333 & 0.0167 & 0.2284 & 0.0216 & 0.2450 & 0.0050 \\
\hline 1 & 0.2369 & 0.0131 & 0.2326 & 0.0174 & 0.2466 & 0.0034 \\
\hline 1.1 & 0.2397 & 0.0103 & 0.2359 & 0.0141 & 0.2476 & 0.0024 \\
\hline 1.2 & 0.2420 & 0.0080 & 0.2387 & 0.0113 & 0.2484 & 0.0016 \\
\hline 1.3 & 0.2437 & 0.0063 & 0.2409 & 0.0091 & 0.2489 & 0.0011 \\
\hline 1.4 & 0.2451 & 0.0049 & 0.2426 & 0.0074 & 0.2492 & 0.0008 \\
\hline 1.5 & 0.2461 & 0.0039 & 0.2440 & 0.0060 & 0.2495 & 0.0005 \\
\hline 1.6 & 0.2470 & 0.0030 & 0.2452 & 0.0048 & 0.2496 & 0.0004 \\
\hline 1.7 & 0.2476 & 0.0024 & 0.2461 & 0.0039 & 0.2498 & 0.0002 \\
\hline 1.8 & 0.2481 & 0.0019 & 0.2469 & 0.0031 & 0.2498 & 0.0002 \\
\hline 1.9 & 0.2485 & 0.0015 & 0.2475 & 0.0025 & 0.2499 & 0.0001 \\
\hline 2 & 0.2488 & 0.0012 & 0.2482 & 0.0018 & 0.2499 & 0.0001 \\
\hline
\end{tabular}


Table 3 : we chose $a=0.45, b=0, H=0.8, \Delta t=0.1$

\begin{tabular}{|c|c|c|c|c|c|c|}
\hline & \multicolumn{4}{|c|}{ The finite difference method [7] } & \multicolumn{2}{|c|}{ The finite element method } \\
\hline $\bar{t}$ & Explicit & $\begin{array}{c}\text { Absolute } \\
\text { enror }\end{array}$ & $\begin{array}{l}\text { Crank- } \\
\text { Nicolson }\end{array}$ & $\begin{array}{l}\text { Absolute } \\
\text { enror }\end{array}$ & $\begin{array}{c}\text { Crank- } \\
\text { Nicolson }\end{array}$ & $\begin{array}{l}\text { Absolute } \\
\text { enror }\end{array}$ \\
\hline 0 & 0.8000 & 0.8000 & 0.8000 & 0.8000 & 0.8000 & 0.8000 \\
\hline 0.1 & 0.6456 & 0.6456 & 0.6517 & 0.6517 & 0.5645 & 0.5645 \\
\hline 0.2 & 0.4885 & 0.4885 & 0.5212 & 0.5212 & 0.3829 & 0.3829 \\
\hline 0.3 & 0.3761 & 0.3761 & 0.4126 & 0.4126 & 0.2606 & 0.2606 \\
\hline 0.4 & 0.2834 & 0.2834 & 0.3238 & 0.3238 & 0.1770 & 0.1770 \\
\hline 0.5 & 0.2127 & 0.2127 & 0.2522 & 0.2522 & 0.1201 & 0.1201 \\
\hline 0.6 & 0.1580 & 0.1580 & 0.1950 & 0.1950 & 0.0815 & 0.0815 \\
\hline 0.7 & 0.1166 & 0.1166 & 0.1499 & 0.1499 & 0.0552 & 0.0552 \\
\hline 0.8 & 0.0855 & 0.0855 & 0.1146 & 0.1146 & 0.0374 & 0.0374 \\
\hline 0.9 & 0.0625 & 0.0625 & 0.0873 & 0.0873 & 0.0253 & 0.0253 \\
\hline$\overline{1}$ & 0.0455 & 0.0455 & 0.0662 & 0.0662 & 0.0171 & 0.0171 \\
\hline 1.1 & 0.0330 & 0.0330 & 0.0501 & 0.0501 & 0.0116 & 0.0116 \\
\hline 1.2 & 0.0239 & 0.0239 & 0.0378 & 0.0378 & 0.0079 & 0.0079 \\
\hline 1.3 & 0.0173 & 0.0173 & 0.0285 & 0.0285 & 0.0053 & 0.0053 \\
\hline 1.4 & 0.0125 & 0.0125 & 0.0215 & 0.0215 & 0.0036 & 0.0036 \\
\hline 1.5 & 0.0090 & 0.0090 & 0.0162 & 0.0162 & 0.0024 & 0.0024 \\
\hline 1.6 & 0.0065 & 0.0065 & 0.0121 & 0.0121 & 0.0017 & 0.0017 \\
\hline 1.7 & 0.0047 & 0.0047 & 0.0091 & 0.0091 & 0.0011 & 0.0011 \\
\hline 1.8 & 0.0034 & 0.0034 & 0.0068 & 0.0068 & 0.0008 & 0.0008 \\
\hline 1.9 & 0.0024 & 0.0024 & 0.0051 & 0.0051 & 0.0005 & 0.0005 \\
\hline 2 & 0.0018 & 0.0018 & 0.0039 & 0.0039 & 0.0003 & 0.0003 \\
\hline
\end{tabular}




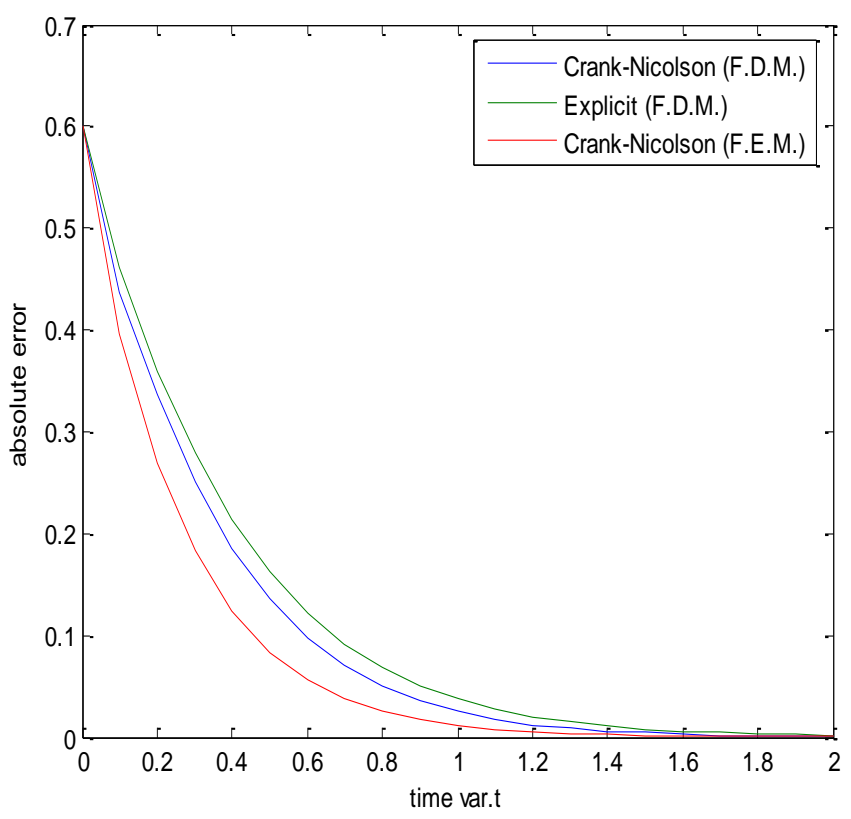

Figure 1:The absolute errors comparison of (F.E.M.)and (F.D.M.) with $\mathrm{a}=0.3, \mathrm{~b}=1, \mathrm{H}=0.2$ and $\Delta t=0.1$

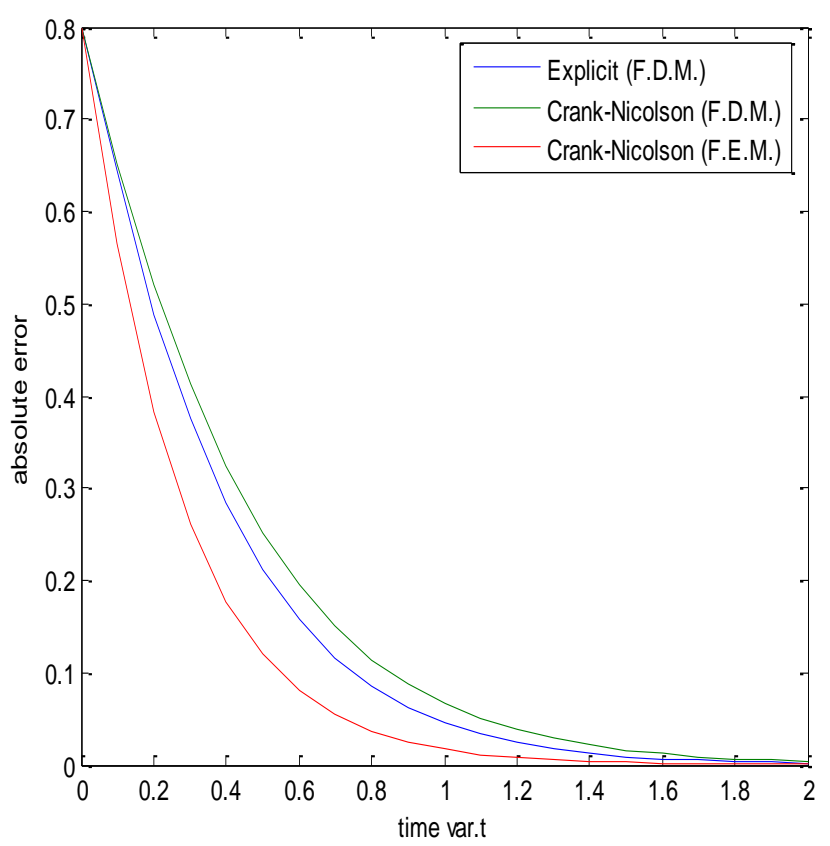

Figure 3:The absolute errors comparison of (F.E.M.)and (F.D.M.) with $\mathrm{a}=0.45, \mathrm{~b}=0, \mathrm{H}=0.8$ and $\Delta \mathbf{t}=\mathbf{0 . 1}$

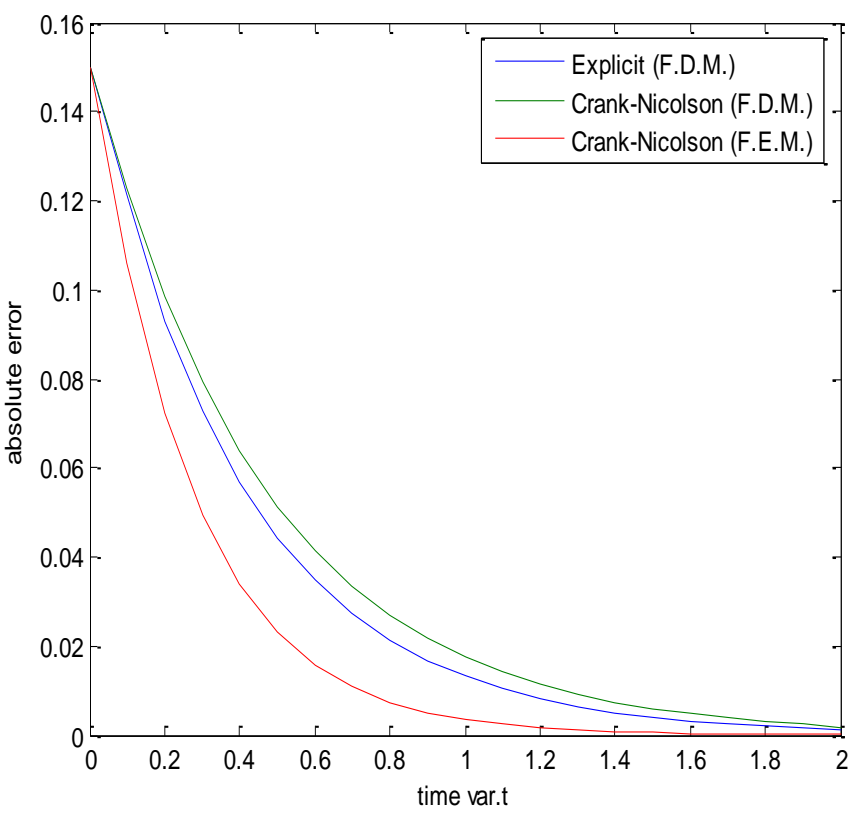

Figure 2:The absolute errors comparison of (F.E.M.)and (F.D.M.) with $\mathrm{a}=0.25, \mathrm{~b}=0.25$, $\mathrm{H}=0.1$ and $\Delta \mathrm{t}=\mathbf{0 . 1}$

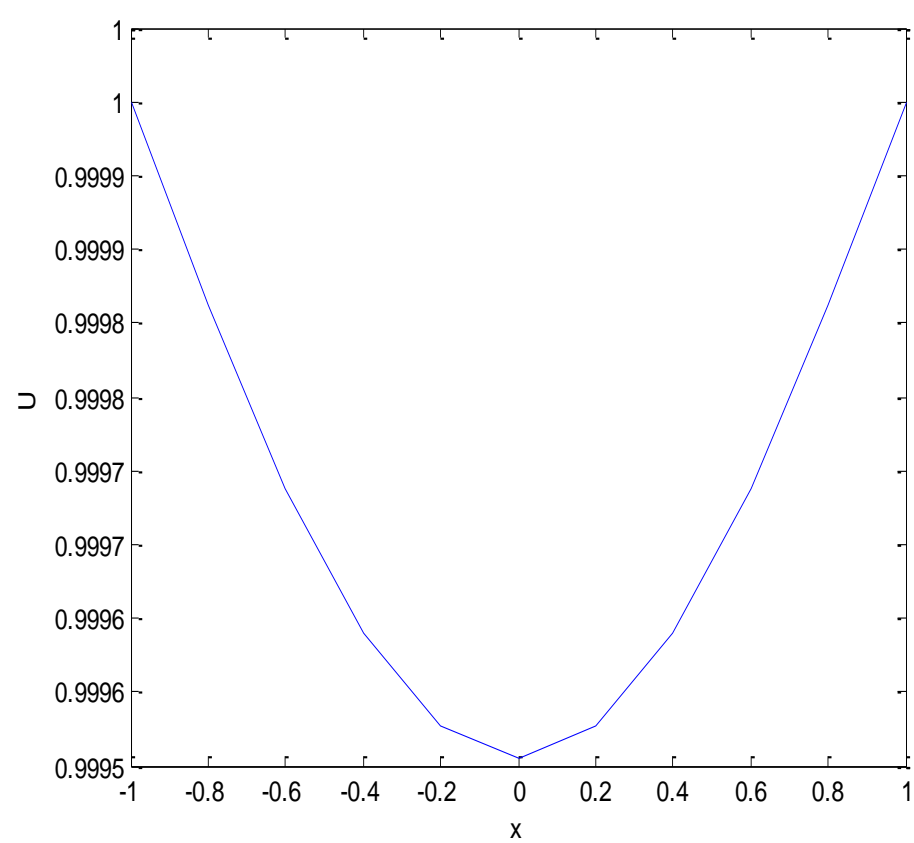

Figure 4: Numerical solution by (F.E.M.) at $\mathrm{a}=0.3, \mathrm{~b}=1, \mathrm{H}=0.2$ and $\Delta \mathrm{t}=\mathbf{0 . 1}$ 


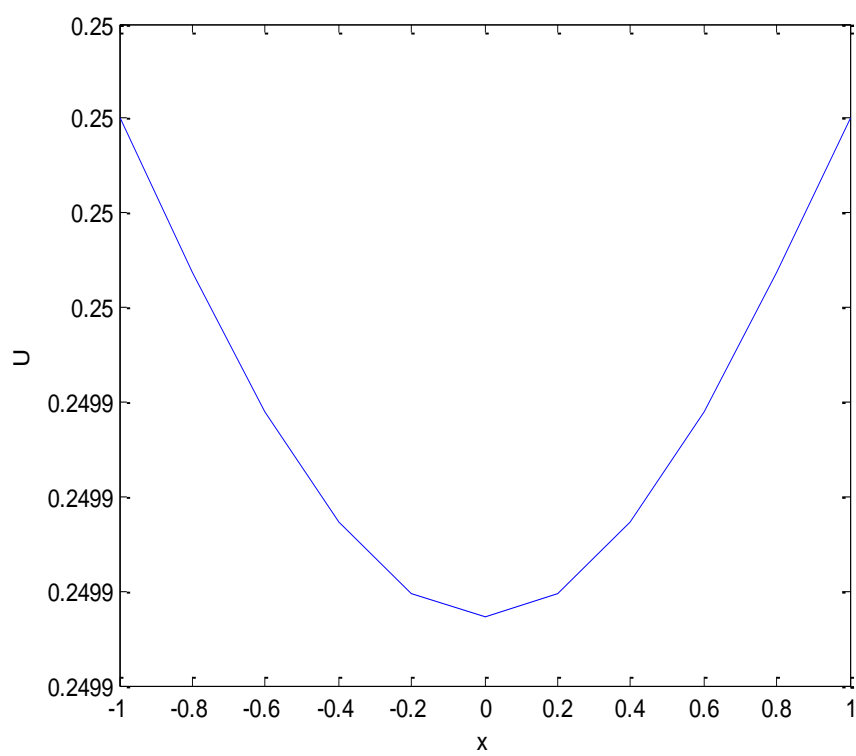

Figure 5: Numerical solution by (F.E.M.) at $\mathrm{a}=0.25, \mathrm{~b}=0.25, \mathrm{H}=0.1$ and $\Delta \mathbf{t}=\mathbf{0 . 1}$

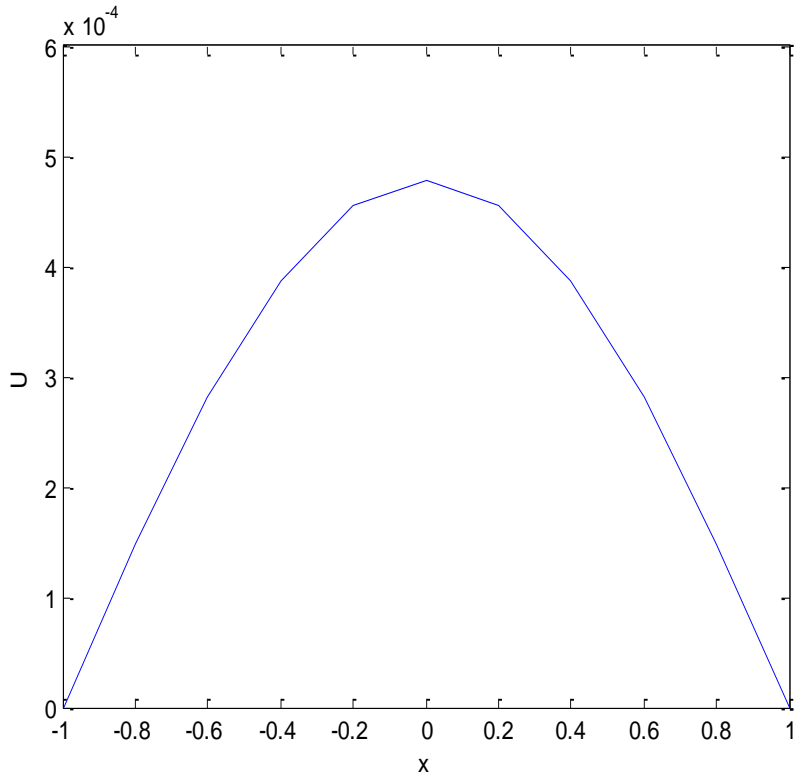

Figure 7 Numerical solution by (F.E.M.) at $\mathrm{a}=0.45, \mathrm{~b}=0, \mathrm{H}=0.8$ and $\boldsymbol{\Delta t}=\mathbf{0 . 1}$

\section{Conclusions}

We have introduced a Crank-Nicolson Galerkin finite element method for solving one dimensional non-linear Huxely equation.The method developed by predictor - corrector method to get on the better results. The numerical results and absolute errors showed that present method is more accurate than the Explicit and Crank-Nicolson finite difference method which represented by [7] .

\section{$\underline{\text { References }}$}

[1] Binczak, S., Eilbeck, J.C. and Scott, A.C., (2001), Ephaptic coupling of myelinated nerve fibers, physica D148, 159-174.

[2] Clarlce, F., (2000), The taylor series coefficients of the jacobi ellipticfunctions, http://www-maths.swan.ac.uk/staff/fwc.

[3] D., A., Frank and Ya., B., Zeldovich, (1938), The theory of thermal propagation of flame, 12, 100-105.

[4] Maginu, K., (1978), Stability of Stationary Solutions of a Semilinear Parabolic Partial Differential Equation, J.Math. Anal. Appl. 63, 224-243.

[5] Manoranjan, V.S., Mitchell, A.R., Sleeman, B.D. and Yu, K. P.(1984),Bifurcation Studies in Reaction-Diffusion, J. Comput. Appl.Math., 11, 27-37.

[6] Manoranjan, V.S.,(1984), Bifurcation Studies in Reaction-Diffusion II, J.Comput. Appl. Math., $11,307-314$. 
[7] Mohmaad Abd, University of Mosuel, College of Computers Sciences and Mathematics, Stability Analysis and Numerical solution of huxely equation, Master Thesis, (2005).

[8] M., K., Jain, (1984), Numerical Solution of Differential Equations, Department of mathematics, Indian Institute of Technology, Delhi.

[9] Thomee, V., (1984), Galerkin finite element for parapolic proplem,Springer Verlag, Berlin.

\section{طريقة العناصر المحددة لحل معادلة Huxely اللا خطية}

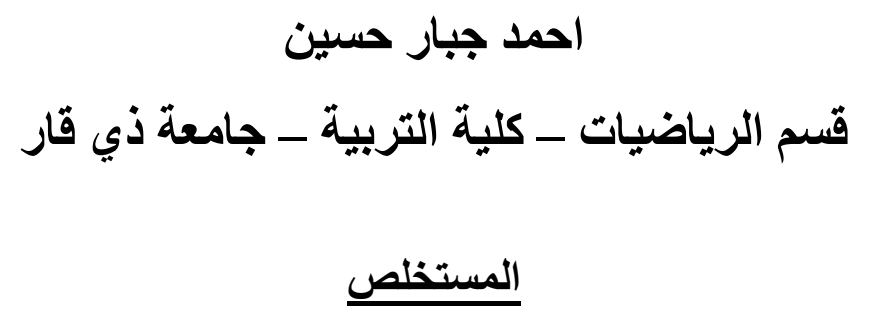

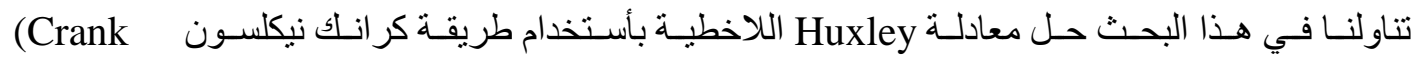
(predictor - corrector مع طريقة المخمن - المصحح (Finite element) للعناصـر المحددة Nicolson) ( النتائج العددية و الاخطاء المطلقة المبينة في هذا البحث هي اكثر دقة و افضل من النتائج العدديـة الممثلة بأستخدام الطريقة الصـريحة (Explicit) وكر انك نيكلسون (Crank Nicolson) للفروقات المنتهيـة عinite) . [7] difference) 